\title{
Study of Centralized Anti-Islanding Method on Large-Scale Photovoltaic Power Plants
}

\author{
Chen-Xin Liu ${ }^{1, a}$, Cheng-Hong Tang ${ }^{1, b}$ \\ ${ }^{1}$ NARI Technology Development Limited Company, Nanjing 211106, China. \\ achenxinleo@163.com, btangchenghong@sgepri.sgcc.com.cn
}

Keywords: Islanding detection, Centralized anti-islanding Method, High-frequency reactance

\begin{abstract}
This paper presents a centralized anti-islanding method on large-scale PV power plants. Contrast to traditional perturbation technology based on single photovoltaic inverter, this method employs a centralized protection device to inject current disturbance onto the photovoltaic power plants, and the measurements of the high-frequency reactance is used to identify islanding. Defects of active islanding detection method for multiply parallel photovoltaic inverters are analyzed. Simulation results demonstrate the effectiveness of the technique for islanding identification of large-scale photovoltaic power plants.
\end{abstract}

\section{Introduction}

Environmental disruption of traditional energy caused an increased application of large-scale renewable power plants. Integration of solar and wind energy to power system has remarkable influences, including increased reliability, reduced line losses and peak-frequency modulation. On the other hand, some problems may be generated. One of the most critical concerns is islanding detection. Unintentional islanding is a condition in which a portion of the distribution network comprising local loads and one or more distributed generation (DG) systems remain energized while isolated from power system. Based on IEEE Std. 929-2000 and IEEE Std. 1547-2003 [1, 2], an unintentional island should be detected within 2s. Therefore, a fast and accurate islanding detection method is required.

At present, the anti-islanding protection of photovoltaic (PV) power plants mainly depends on PV inverter. The grid-connected inverter determines whether the islanding occurs by injecting perturbations, then detects whether the outlet voltage and current signal exceed a predetermined threshold. However, power quality deterioration and increased dead zone may result from a large number of inverters working at the same time. Furthermore, there are technical difficulties that achieving anti-islanding protection and low voltage ride through (LVRT) in the same PV inverter.

In this paper, a centralized anti-islanding method based on both passive and active methods is presented, that is accomplished by a dedicated anti-islanding protection device on large-scale renewable power plants. The proposed method is based on high-frequency reactance calculation, which is a better scheme than above mentioned methods when applied on large-scale renewable power plants.

\section{Interactions between parallel PV inverters}

Large-scale PV power plants are generally composed of dozens of parallel connected PV power generation, which contains a large number of reactive power consuming equipment, i.e. the low-voltage transformer, low-voltage cables, main transformer, etc. Thus, reactive power compensation equipment is installed to satisfy the voltage amplitude and power factor specifications. The cost of large-capacity Static Var Generator (SVG) is fairly high. Therefore, the reactive power required by PV power plant is provided by parallel capacitor and SVG. The schematic of grid-connected PV power plant is showed in figure 1. 


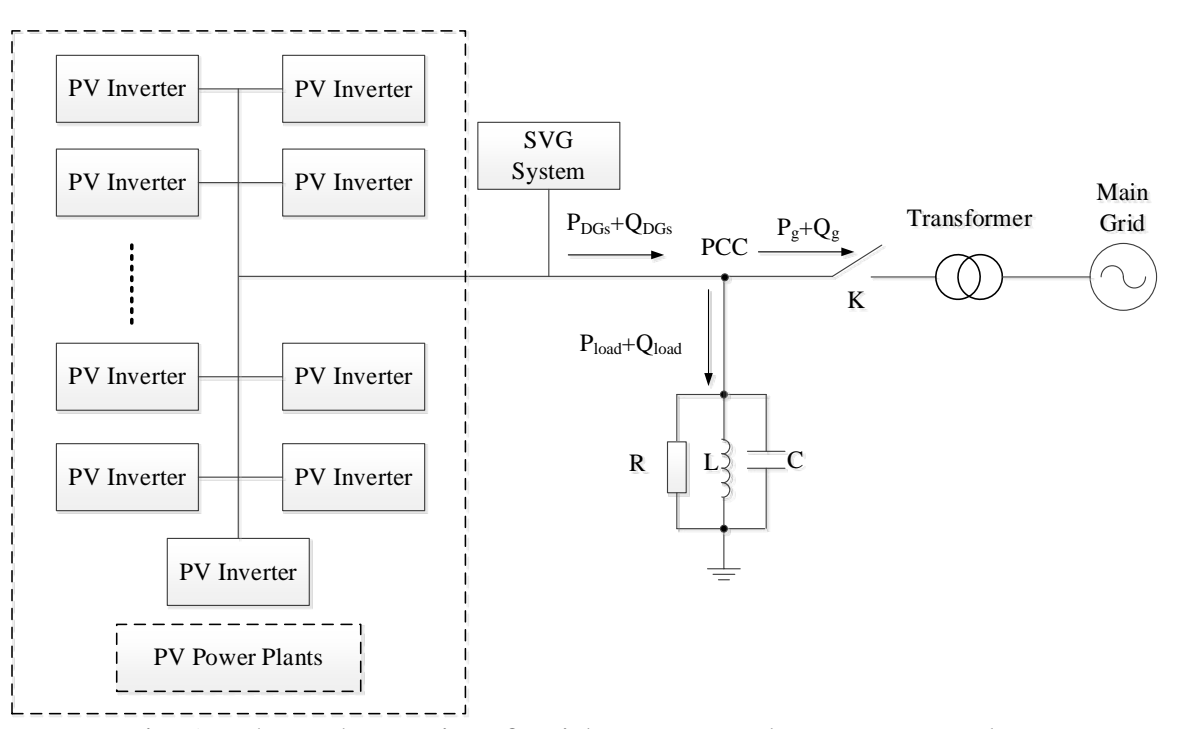

Fig 1. The schematic of grid-connected PV power plant

The parallel connected PV inverters are independent of each other. The traditional active anti-islanding methods introduce disturbances into grid from single PV inverter output, which amplify the changes of magnitude or frequency. The output of each inverter needs to provide a perturbation according to its corresponding control strategy. Different types of perturbation superimpositions are introduced, due to the PV inverters, which commonly come from different manufacturers in large-scale PV power plants. Therefore, the following problems may be present:

- Disturbance of different types is diluted. The corresponding electrical quantity cannot exceed the threshold, resulting in multi-device islanding detecting failure.

- Disturbance of same types is weak. The disturbance quantity cannot make the corresponding electrical quantity exceeding threshold value, resulting in islanding detecting failure.

- When the disturbance of the same type is strong, the power superimposed by dozens of inverters may cause significant changes of the voltage amplitude or frequency, which produce power quality problems, moreover, cause protection malfunction.

In large-scale PV power plants, the traditional distributed active islanding detecting strategy based on separated inverters may lead to anti-islanding failure and deterioration of power quality due to the inconsistencies of the disturbed perturbation signals.

\section{Failure analysis of active islanding detection method based on single PV inverter}

Active islanding detection methods commonly make use of variations of reactive power or frequency accomplished by a PV inverter. The perturbations purposely injected may break the power balance between the DG and the local loads. Reactive-frequency feedback method is used as an example to illustrate the defect of active islanding detection method based on single PV inverter.

Reactive-frequency feedback method is based on measured frequency difference between grid and point of common coupling (PPC), which controls the inverter to generate specific reactive power. In grid-connected operation, the two frequencies are close to each other, thus the reactive power output is approximately zero. In islanding operation, the generated reactive power is amplified by the feedback coefficient, controlled by PV converter depending on the frequency difference. The control algorithm is:

$$
Q_{\text {inv }}=k\left(f_{a}-f_{g}\right)
$$

$Q_{i n v}$ is the reactive power output by the inverter, $k$ is the frequency feedback coefficient, $f_{a}$ is the measured frequency of main grid, and $f_{g}$ is rated frequency.

The frequency of PCC will only have a small offset even if the islanding power matching degree is high. The PV inverter will output a certain reactive power, resulting in an increasing reactive power 
mismatch under the action of the feedback coefficient. Then the frequency quickly exceeds the threshold. However, this method may have NDZ when the PV inverters running in parallel.

\section{Centralized Anti-Islanding Method}

Active islanding detection methods commonly make use of variations of reactive power or frequency accomplished by a PV inverter. The perturbations purposely injected may break the power balance between the DG and the local loads. Reactive-frequency feedback method is used as an example to illustrate the defect of active islanding detection method based on single PV inverter.

Main principle. Unified disturbance signals are injected into grid from PPC by centralized injection device, which avoids the interference between multiply inverters. The overall scheme is showed in figure 2. The inverter disturbance mode is turned off. Yet the centralized disturbance mode independent of each power source is installed. The system has only one unified source of disturbance. The generation and injection of the disturbance are controlled by concentrated injection device, which is independent of the inverter.

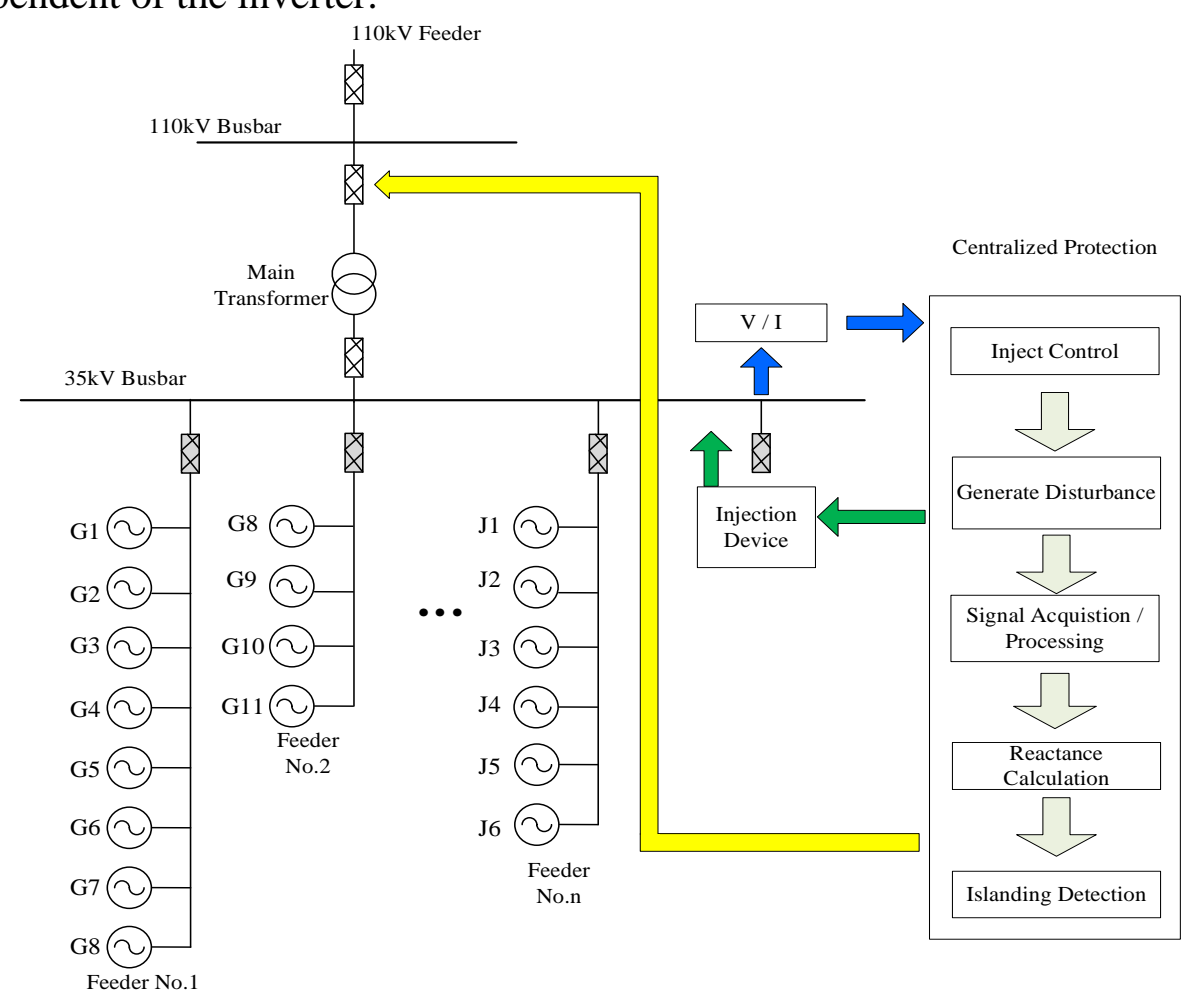

Fig 2. The diagram of reactive-frequency feedback method

The proposed method can be applied to multi-inverter power supply system. The centralized device injects the disturbance signal into the system. The response of the system is collected during the injection process by centralized protection device through monitoring the zero-crossing point of PPC. The high-frequency reactance is used to amplify the characteristic difference. Furthermore, the islanding state identification is performed by certain logic criterions.

Perturbation Injection Method. The perturbation source is accomplished by single-phase full-bridge inverting circuit, which is connected with a large coupling inductor. The value of the coupling inductance is related to the voltage and the required disturbance current. The grid-connected switch controls the connection between the disturbance generator and the system.

A pulse of triangle current wave is generated from the coupling inductance through the breaking changes of IGBT. The pulse width is controlled by the pulse width of the disturbing current signal. Yet the value of the DC voltage and the coupling inductance $L$ is used to control the peak value of the disturbance current signal. The perturbation injection signal is intermittent, which can reduce the disturbance to the system than the specific frequency harmonic continuous injection strategy. The 
waveform is showed as figure 3 . The frequency spectrum of injected perturbation is showed as figure 4, which contains continuous frequency components.

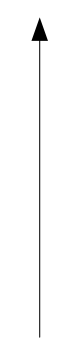

Fig 3. Voltage and current disturbance signal wave

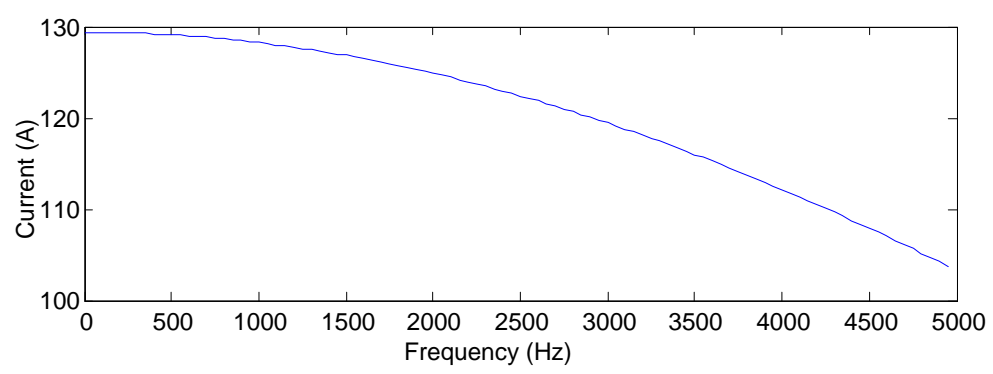

Fig 4. Frequency spectrum of injected perturbation

A zero-crossing injection method is used as the main injection strategy by monitoring the voltage of PCC, through which the pulse of triangle current wave is injected at each zero-crossing point. The dynamic zero-crossing injection method can improve the signal-to-noise ratio and the accuracy of the impedance analysis calculation, which is realized by maximizing the voltage component of the original system. Furthermore, zero-crossing injection method can reduce the required disturbance injection level, which is unlike traditional specific frequency harmonics that require sustained injection.

High-Frequency Reactance Measurement Algorithm. High-Frequency reactance measurement methods for islanding detection are all based on the change of reactance as viewed from the injection device when islanding occurs. In general, the reactance increases after islanding forms. Indirect methods inject a high frequency signal through a triangular current signal. This high frequency signal is expected to be more significant after the grid is disconnected. Direct methods directly measure the reactance by injecting a controlled disturbance. For centralized injection method, a large change in current is required to evaluate the reactance changes.

High-frequency reactance is used as the feature quantity of the isolated islanding, which improves the accuracy and sensitivity of the measurement. The difference between the characteristic values before and after islanding is amplified by the high frequency level, which greatly weakens capacitive reactance. Thus better linear relationship of frequency-domain is achieved, which is used to calculate the average of specific frequency reactance, rather than calculating directly. The triangular current signal wave injected into the system is rich in high frequency components. Assuming that the system impedance changes linearly in the frequency domain, the system is equivalent to short-circuit. High-frequency reactance of the system can use the time-frequency domain transformation measurement.

\section{Simulation of proposed method}

A large-scale multiply PV parallel system is established to verify the reliability of centralized injection perturbation method based on high-frequency reactance measurement. Different control strategies are used based on different $K_{\mathrm{p}}$ and $K_{\mathrm{i}}$ parameters. The output of PMSGs is set as 0.9 times 
to rated power respectively, which is matched perfectly to the local load. The islanding occurs at 5s, while the total simulation time is 10 s. The results are showed as figure 5.

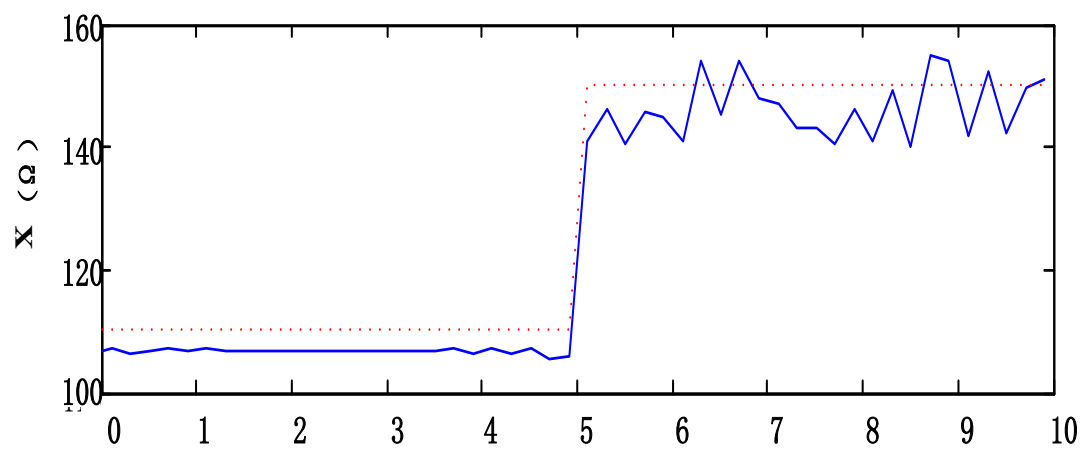

Fig 5. High-frequency reactance measurement in time domain

The result shows that the change of high-frequency reactance is $34.1 \%$, which is higher than the set threshold value of $15 \%$. The proposed method can quickly and reliably detect islanding statement. For testing the impact of the proposed method on the power quality of the system, the harmonic content is compared with the figure 6. Blue solid line is the voltage harmonic of PCC when no injection, while red dotted line is for the injection signal. For facilitating harmonic contrast, $50 \mathrm{~Hz}$ fundamental component content has been removed.

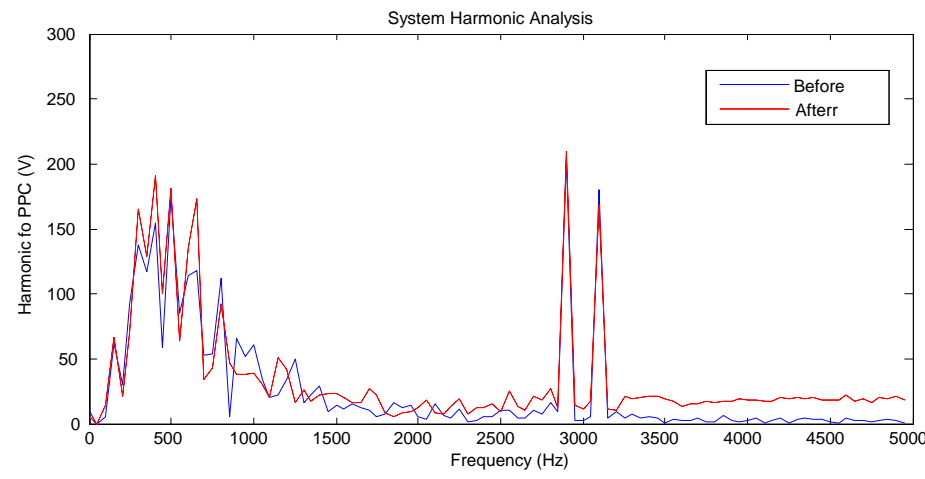

Fig 6. Analysis of system harmonic before and after injection

The voltage harmonics are mainly concentrated in the low frequency nearly $3 \mathrm{k} \mathrm{Hz}$ without injection, which is electronic devices switching frequency approximately. After the disturbance signal is injected, the harmonic content is slightly increased between lower frequency band (around $500 \mathrm{~Hz}$ ) and high frequency band (greater than 3.5k Hz). However the impact on THD is not significant, which proves that the proposed method does not affect the system Power quality during normal operation.

\section{Conclusion}

In this paper, a new anti-islanding technique has been demonstrated both practically and in simulation. The failure of traditional active islanding detection method based on single PV inverter is analyzed. The high-frequency reactance is applied to perturbation calculation, which is used for centralized anti-islanding detection. Simulation results demonstrate the effectiveness of the technique for islanding identification of large-scale PV power plants.

\section{Acknowledgments}

This work was financially supported by State Grid Corporation of China for "Study and Application of Centralized Anti-Islanding on Large-Scale renewable energy” project. 


\section{References}

[1] IEEE Recommended Practice for Utility Interface of Photovoltaic (PV) Systems, IEEE Standard 929, 2000.

[2] IEEE Standard for Interconnecting Distributed Resources with Electric PowerSystems, IEEE Standard 1547, 2003.

[3] R. M. Rifaat. "Critical considerations for utility/cogeneration inter-tieprotection scheme configuration" IEEE Trans. Industry Applrcations, vol.31, no.5. pp.973-977. Sep/Oct.1995.

[4] C. S. Morina. "Interconnection protection of IPP generators at commercial/industrial facilities" IEEE Trans. Indusrry Apphcalions, vol.37, no.3, pp.681-687. May/June 2001.

[5] A.M. Masoud, K.H. Ahmed, S.J. Finney, B.W. Williams, Harmonic distortion-based island detection technique for inverter-based distributed generation, IET Renewable Power Generation 3 (4) (2009) 493-507.

[6] G. K. Hung, C. C. Chang, C. L. Chen, "Automatic phase-shift method for islanding detection of grid-connected photovoltaic inverters," IEEE transactions on energy conversion, Vol. 18, No.1, pp. 169-173, March 2003.

[7] J. Stevens, R. Bonn, J. Ginn, S. Gonzales, "Development and testing of an approach to anti-islanding in grid interconnected photovoltaic systems," Sandia National Laboratories, Albuquerque, NM, Report SAND2000-1939, August 2000.

[8] NERL/SR-560-36243, "Study and development of anti-islanding control for grid connected inverters," Tech. Rep.NAD-1-30605-01, May 2004.

[9] Nowak S. Trends in photovoltaic applications --survey report of selected IEA countries between 1992 and 2006. in Report IEA-PVPS T1-16, 2007

[10] Ranade S. J., Sagi D. R., Mulpuri R., et al. Likelihood of Islanding in Distribution Feeders with Photovoltaic Generation in Power Engineering Society General Meeting, 2007: 1-6 